\title{
Evaporation of Microdroplets of Azeotropic Liquids
}

\author{
S. M. Rowan,* M. I. Newton, F. W. Driewer, and G. McHale \\ Department of Chemistry and Physics, The Nottingham Trent University, Clifton Lane, \\ Nottingham, NG11 8NS, U.K.
}

Received: March 10, 2000; In Final Form: June 12, 2000

\begin{abstract}
This work reports data showing the evolution of contact angle with time for mixtures of water and 1-propanol at room temperature on poly(methyl methacrylate) (PMMA). The composition range investigated spans the azeotropic composition, thus encompassing systems containing excess water and excess 1-propanol. A discontinuity in the contact angle behavior is observed and it is suggested that this enables the identification of the azeotropic composition as 0.39 mole fraction of 1-propanol. This suggestion is supported by boiling point measurements made at around $20 \mathrm{mmHg}$. The discontinuity is associated with the presence of an instability, which causes a distortion around the droplet perimeter. It is suggested that the distortion is caused by competing effects of local surface tension maxima and minima.
\end{abstract}

\section{Introduction}

When a liquid droplet is introduced on to a plane surface it may spread out and wet the surface with a zero angle of contact or it may establish a finite equilibrium contact angle. Additional considerations apply when the liquid is volatile. The evaporation of small droplets of volatile pure liquids from solid surfaces depends on the initial contact angle being greater or less than ninety degrees. ${ }^{1,2}$ In the former case, the initial stage is dominated by the contact angle remaining constant while the contact radius decreases. In the latter case, the contact angle decreases and the contact radius remains constant. The influence of the evaporation of liquid droplets on contact angle measurements has also been reported by other investigators during the past decade, e.g., see refs 3-6.

The front of a pure wetting liquid, as it advances over a surface, is pushed initially from behind by the hydrostatic head of the liquid. As the drop height reduces, this becomes unimportant and further wetting has been shown ${ }^{7}$ to be associated with the presence of unbalanced forces operating on the liquid front. These forces cause a periodic shape to develop in the liquid front as it advances, indicating the presence of an instability. Known instances of instabilities in fluids include Rayleigh-Taylor instability that arises when two immiscible liquids are stratified with the denser fluid above and the Marangoni effect, which is associated with temperature and concentration gradients. Consequently, if a liquid mixture is present in a surface droplet, then Marangoni forces arising from concentration gradients are likely to be present during evaporation. The use of liquid mixtures in wetting studies has been reported in the literature, e.g., ${ }^{8}$ in order to apply the methods of Zisman ${ }^{9}$ and Fowkes. ${ }^{10}$ In their approaches, liquid mixtures of different composition are used to provide a range of contact angle values and surface tension on a given surface. For example, liquid mixtures based on ethylene glycol and water enable the surface tension to be varied systematically between 48 and $72 \mathrm{mN} \mathrm{m}^{-1}$ at $20^{\circ} \mathrm{C}$ It is therefore of general interest to know what will happen to contact angle development with

* Author to whom correspondence should be addressed. Tel: +44 (0)115 8483218. Fax: +44 (0)115 9486636. E-mail: mike.rowan@ntu.ac.uk. time if both components of a liquid mixture are volatile. Such mixtures are likely to form nonideal solutions whose deviations from Raoult's Law lead to the formation of either a low- or a high-boiling azeotrope. A solution which shows sufficiently positive deviations from Raoult's Law gives rise to an azeotrope with a minimum boiling point. When such a mixture containing one or other of the components in excess of the azeotropic composition is evaporated, a residual liquid consisting of the pure component in excess is produced.

Water and 1-propanol form an azeotrope with a normal minimum boiling point of $87.7^{\circ} \mathrm{C}$ and a composition of 0.4 mole fraction of 1-propanol. ${ }^{11}$ In principle the composition of an azeotrope may vary with pressure, although the data presented in International Critical Tables ${ }^{11}$ show no such change between 700 and $800 \mathrm{mmHg}$. It is likely that any change in azeotropic composition over the range $10-700 \mathrm{mmHg}$ will be small. Pure 1-propanol wets poly(methyl methacrylate) (PMMA), while in contrast pure water does not; a droplet of pure water on PMMA produces an advancing contact angle of $59.3^{\circ 12}$ at $20{ }^{\circ} \mathrm{C}$. In this work, we report data for the evolution of contact angle with time for mixtures of water and 1-propanol at room temperature on PMMA. The composition range investigated spans the azeotropic composition, thus encompassing systems containing excess water and excess propanol. A discontinuity in the contact angle behavior is observed, and it is suggested that this enables the identification of the azeotropic composition. This interpretation is supported by boiling point measurements made at around $20 \mathrm{mmHg}$.

\section{Experimental Section}

Liquid mixtures of triply distilled water and $99.5 \%$ pure 1-propanol were prepared with compositions of 75, 70, 67.5, 65,60 , and 55 by percentage weight. These correspond to mole fractions of 1-propanol of $0.47,0.41,0.39,0.36,0.31$, and 0.27 . Contact angle experiments were performed using droplets of these mixtures deposited onto a flat surface of PMMA with deposition from a syringe brought lightly into contact with the substrate. Two separate contact angle measurement systems were used in order to examine the full range of experiments. In the first system, two cameras were mounted such that both a plan 


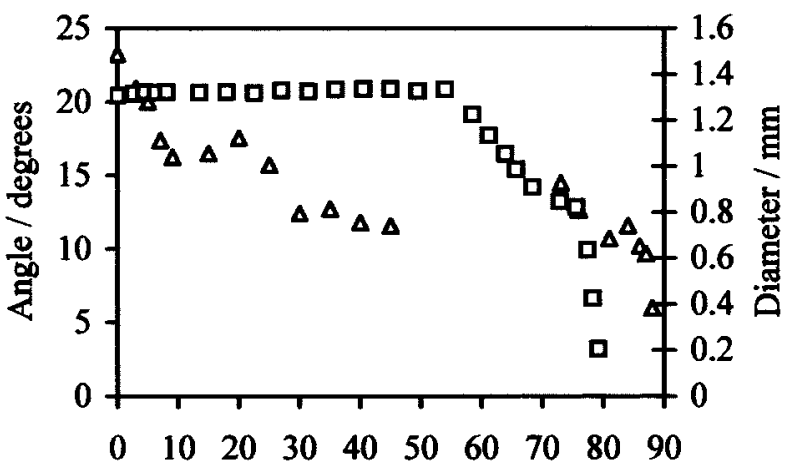

Time / seconds

Figure 1. The evolution of the contact angle and base diameter of a small droplet of water-propanol mixture $(0.47$ mole fraction of propanol) from a PMMA substrate.

view and a profile view of an evaporating droplet could be observed simultaneously during an experiment. This was particularly useful to observe the development of instabilities around the perimeter of a droplet. The second system used a Krüss contact angle meter to view droplets in profile. The profiles were recorded onto videotape and were then captured onto a personal computer using a Data Translation DT3512 scientific framegrabber card. The optical/capture system had vertical and horizontal calibrations of 422.8 and 439.8 pixels per $\mathrm{mm}$, respectively, and a $768 \times 576$ pixel field of view. All data presented within this paper were taken using this second system. The temperature for these experiments was maintained at $21{ }^{\circ} \mathrm{C}$ to within $1{ }^{\circ} \mathrm{C}$, although the ambient relative humidity varied slightly between experiments. Liquid boiling point measurements were performed to provide an estimate of the azeotropic composition at $20 \mathrm{mmHg}$. These measurements were conducted under vacuum in a reflux apparatus fitted with a pressure transducer and an electronic thermometer.

\section{Results and Discussion}

In the following discussion 1-propanol is abbreviated to propanol. Two distinctly different types of behavior were observed for droplet evaporation, depending on whether the composition of the mixture exceeded 0.39 mole fraction of propanol or was less than 0.36 ; a transition region existing between these two mole fractions. When the mole fraction of propanol exceeded 0.39 , droplets showed initial contact angles within the range $16-28^{\circ}$. However the variation was not systematic with composition. For any single droplet, the contact angle decreased steadily over a period of about 90 s which was required for complete evaporation, the base diameter remained constant for approximately the first 60s, before decreasing rapidly. Figure 1 illustrates the evolution of contact angle and base diameter in the case of 0.47 mole fraction of propanol. This behavior, in particular the pinning of the contact line of the droplet, is similar to that observed when a small drop of water, i.e., a single pure component, is allowed to evaporate after deposition on $\mathrm{PMMA}^{2}$. A sequence of images showing the droplet profile and its reflection for these data is given in Figure 2. The droplet has an approximately spherical cap-type shape throughout the period of evaporation. There is some indication from the profiles that when the contact line of the droplet de-pins, the droplet continues to sit on a wider residual film. It is also possible to see a change in contrast directly above the droplet, and this is probably due to the vapor from the evaporating droplet.

For mole fractions of propanol of 0.36 or less, an initial contact angle of $15-30^{\circ}$ was established. However, the subse-
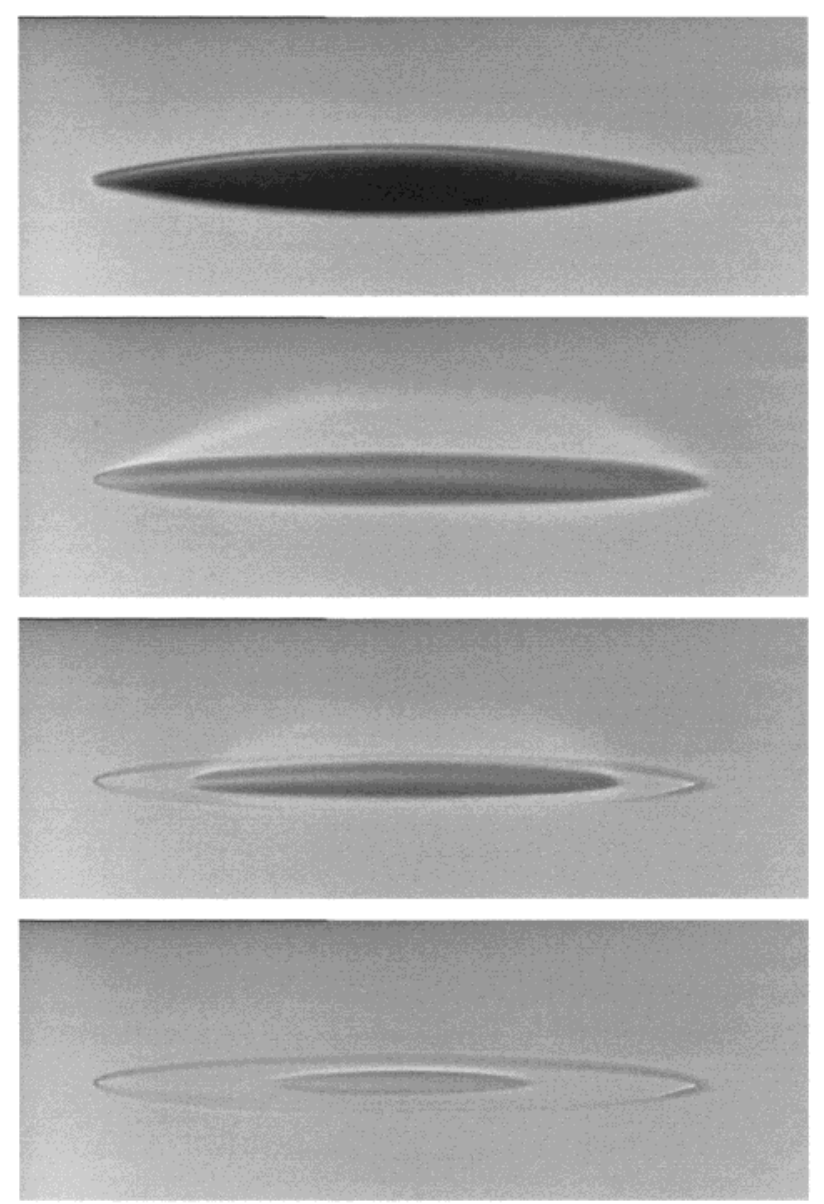

Figure 2. A sequence of images (top to bottom for increasing time) showing the evolution of the droplet profile for the data in Figure 1.

quent behavior differed sharply from that observed when the mole fraction of propanol exceeded 0.39. Following a short period during which the contact angle decreased, there was a period of time in which the angle could not be measured due to instability of the droplet around its periphery. This instability provides the greatest contrast in behavior for droplets whose initial mole fraction of propanol is 0.36 or less from those whose mole fraction exceeds 0.39 . In the case of a droplet of 0.36 mole fraction of propanol, a peripheral distortion sets in and is maintained until the droplet breaks up into several small droplets. For mole fractions of propanol smaller than 0.36 , the period of instability is usually concluded by the formation of a single new droplet. In some experiments the initial stage of spreading and evaporation leads to an apparent complete disappearance of the droplet as viewed in profile, only for a new, residual, droplet suddenly to re-appear moments later. Figure 3 shows a droplet of 0.31 mole fraction of propanol and illustrates the instability and subsequent formation of a single new droplet. Generally, as a new droplet developed, the contact angle increased from around $10^{\circ}$ to reach a maximum of $35-45^{\circ}$ and then subsequently decreased, as for a single component drop. After the formation of the new droplet, the base diameter decreased with time as evaporation proceeded.

Figures 4 and 5 illustrate contact angle and base diameter evolution for 0.31 and 0.27 mole fractions of propanol, respectively. For mole fractions of propanol less than 0.36 , the formation of a new, residual droplet is consistent with the presence of a single pure liquid that is nonwetting to PMMA. It is therefore reasonable to suggest that in this case the residual droplet is water, since pure propanol wets PMMA. A pure water 

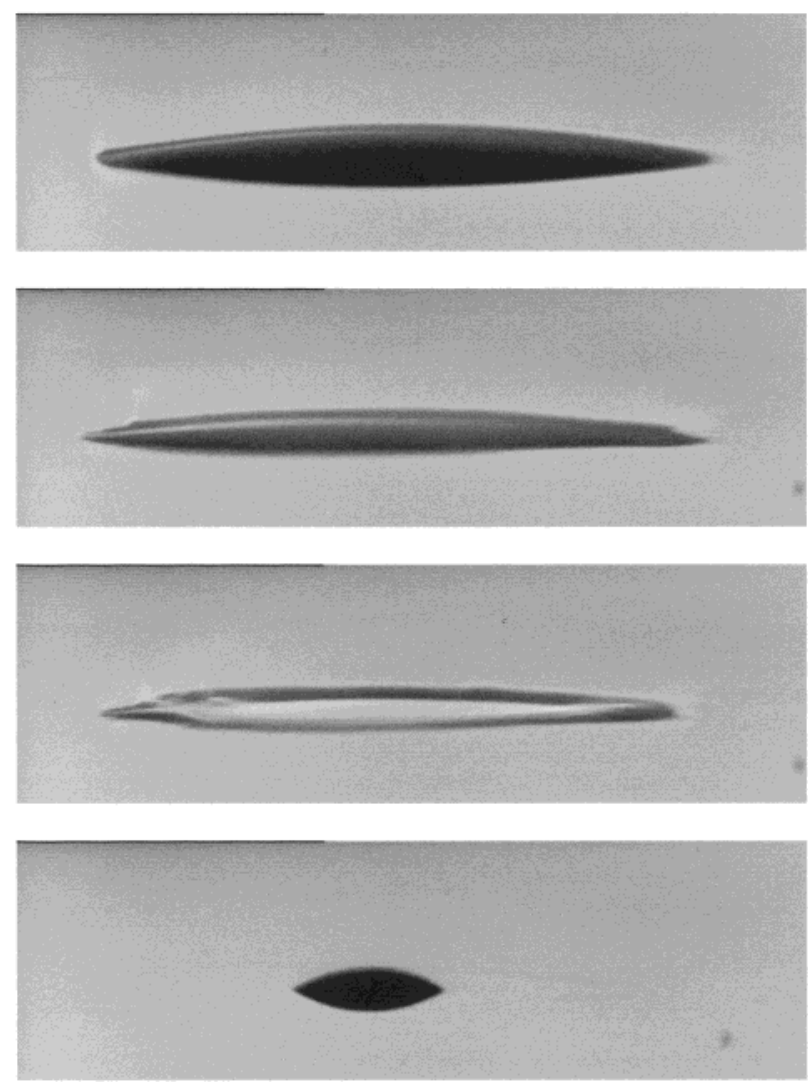

Figure 3. A sequence of images showing the evolution of a droplet profile obtained for a mole fraction of 0.31 of propanol. The formation of a single new droplet after a period of instability occurs for mole fractions of propanol of less than 0.36 .

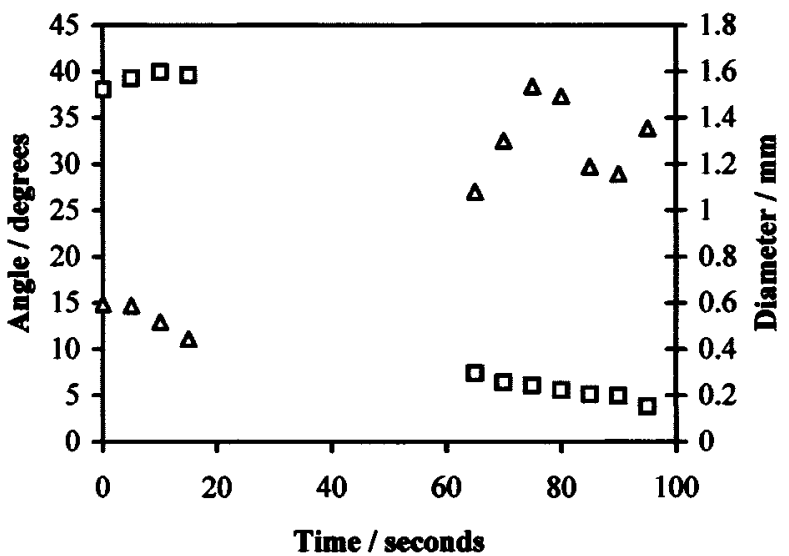

Figure 4. Contact angle and base diameter evolution as a droplet of 0.31 mole fraction of propanol evaporates from a PMMA substrate.

droplet on clean PMMA shows an advancing contact angle of about $60^{\circ}$. This suggestion is consistent with evidence provided from boiling point measurements at a vapor pressure $20 \mathrm{mmHg}$ for a series of mixtures covering the entire range of compositions. These show (Figure 6) that a minimum occurs at a boiling point of $286 \mathrm{~K}$ and provide an estimate of the azeotropic composition as 0.41 mole fraction of propanol. This composition is close to that associated with the droplet transition to instability when the mole fraction of propanol is less than 0.39 .

The evaporation of a mixture with a mole fraction of propanol of less than 0.41, the azeotropic composition, would be expected to leave a residual liquid of pure water. The initial contact angle of the mixtures are much less than the expected value for pure water of $60^{\circ}$, but higher than that expected for pure propanol. Multidroplet formation at 0.36 mole fraction of propanol may

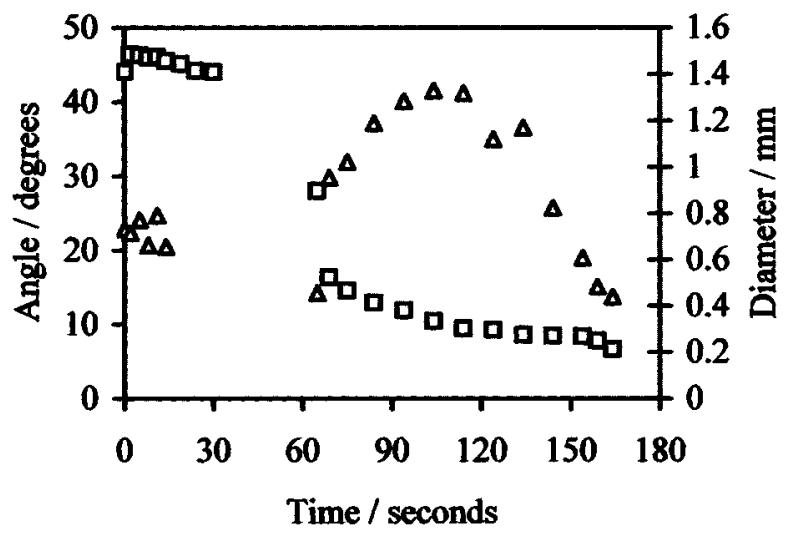

Figure 5. Contact angle and base diameter evolution as a droplet of 0.27 mole fraction of propanol evaporates from a PMMA substrate.

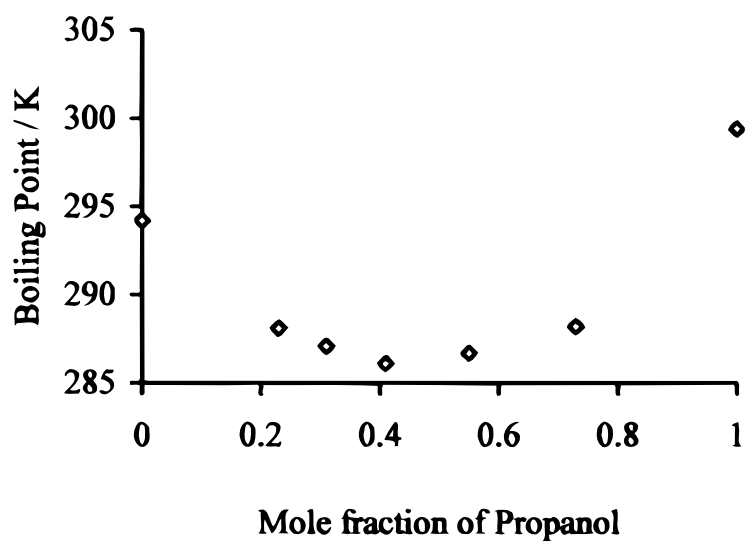

Figure 6. Data from boiling point measurements show the azeotropic composition for water-propanol mixtures at $20 \mathrm{mmHg}$ is at around 0.41 mole fraction of propanol.

be ascribed to an insufficient excess of water close to the azeotropic composition. For compositions where the mole fraction of propanol exceeds that of the azeotrope, the initial period of evaporation will lead to residual liquid of pure propanol, which is wetting to PMMA. As a result of this the contact angle decreases continuously without instability in the droplet. It is suggested that the azeotropic composition will correspond to the lowest propanol mole fraction for which instability and new droplet formation are not observed, i.e., 0.39 in this work. This value agrees, within experimental accuracy, with the estimate of 0.41 established from boiling point measurements.

The origin of the instability observed for droplets of mixtures containing an excess of water over the azeotropic composition may be associated with Marangoni forces arising from surface tension and concentration gradients. The presence of instability suggests two competing effects; one of these could be preferential adsorption of propanol producing local surface tension minima, and the other could be cooling giving surface tension maxima. It can be anticipated that local maxima and minima in the surface tension of evaporating droplets could produce an undulating contact line perimeter. One possible mechanism for generating such local maxima and minima would involve evaporative cooling and preferential adsorption. Evaporation of droplets of water/propanol mixtures produces a vapor that becomes progressively richer in azeotropic composition, while leaving a residual liquid that tends to the pure liquid in excess. The evaporation causes the surface temperature to drop, thus causing the surface tension to increase. Propanol is amphiphilic in character and therefore is adsorbed preferentially to water at 
the liquid-vapor interface, reducing the surface tension as a result. It is conceivable that when water is the liquid in excess in the droplet mixture, a stage in the evaporation will be reached when the remaining propanol will be present in patches on the surface. Hence the combination of propanol patches causing local surface tension minima and cooling producing surface tension maxima will in turn cause the surface to undulate prior to the formation of a new residual droplet of pure water. When propanol is present in excess, local patches of water are less likely to occur in the surface, as water lacks amphiphilic character. Consequently one of the requirements for producing surface undulation is absent and surface instability would not be observed.

\section{Conclusion}

Contact angle measurements and their variation with time have been made for droplets formed from mixtures of water and 1-propanol at $21{ }^{\circ} \mathrm{C}$ on PMMA. Two contrasting types of behavior have been observed when droplets of water/1-propanol evaporate at $21{ }^{\circ} \mathrm{C}$ from the surface of PMMA, depending on whether 1-propanol or water is present as the component in excess in an azeotropic mixture. When 1-propanol was in excess, the contact angle decreased throughout the evaporation and the contact line was pinned for around two-thirds of the evaporation time; the droplet also retained an approximately spherical captype shape. When water was present in excess, the droplet became unstable and was distorted around its perimeter. The original droplet then virtually disappeared only for a new droplet to be subsequently formed. The contact angle of this new droplet increased to a maximum before decreasing as the droplet evaporated. The composition at which the instability and distortion were first observed was 0.39 mole fraction of 1-propanol. It is suggested that this value is a measure of the azeotropic composition. A value of 0.41 mole fraction of 1-propanol for the azeotropic composition has been estimated independently from boiling point measurements. It is suggested that the origin of the droplet instability lies in the competing effects of local surface tension maxima and minima. The former arising from the cooling effect caused by evaporation and the latter from preferential adsorption of 1-propanol at the liquidvapor interface.

Acknowledgment. The authors acknowledge technical assistance from A. Pope and F. Barlerin during the course of this work.

\section{References and Notes}

(1) McHale, G.; Rowan, S. M.; Newton, M. I.; Banerjee. M. K. J. Phys. Chem. 1998, B102, 1964.

(2) Rowan, S. M.; Newton, M. I.; McHale, G. J. Phys. Chem. 1995 99, 13268

(3) Birdi, K. S.; Vu, D. T.; Winter, A. J. Phys. Chem. 1989, 93, 3702.

(4) Birdi, K. S.; Vu, D. T. J. Adhes. Sci. Technol. 1993, 7, 485.

(5) Shanahan, M. E. R.; Bourgès, C. Int. J. Adhes. Adhes. 1994, 14, 201.

(6) Bourgès, C.; Shanahan, M. E. R.; C. R. Acad. Sci. (Paris) 1993, 316,311 .

(7) Williams, R. Nature 1977, 266, 153.

(8) Banczuk, B.; Bialopiotrowicz, T.; Wojcik, W. J. Colloid Interface Sci. 1989, 127, 59.

(9) Fox, H.W.; Zisman, W.A. J. Colloid Interface Sci. 1950, 5, 514.

(10) Fowkes, F. M. Adv. Chem. Ser. 1964, 43, 99.

(11) National Research Council (U.S.). International Critical Tables of Numerical Data, Physics, Chemistry and Technology; McGraw-Hill: New York, 1933; Vol. 3, p 310.

(12) Adamson, A. W.; Gast, A. P. Physical Chemistry of Surfaces, 6th ed.; J.Wiley and Sons: New York, 1997; p 365. 